\title{
Laboratory experiments and observations of cyclonic and anticyclonic eddies impinging on an island
}

\author{
Magdalena Andres ${ }^{1}$ and Claudia Cenedese ${ }^{1}$ \\ Received 7 September 2012; revised 17 December 2012; accepted 9 January 2013; published 13 February 2013.
}

[1] Laboratory experiments are conducted to investigate the interactions of selfpropagating barotropic cyclones and baroclinic anticyclones with an island. Results are interpreted in the context of observations around Okinawa Island, Japan, where ubiquitous arrivals of cyclones and anticyclones on the southeastern side of the island influence the flow around it, thereby impacting both the Ryukyu Current's and the Kuroshio's transport. In the laboratory, baroclinic anticyclones generate a buoyant current that flows clockwise around an island whereas barotropic cyclones generate a counterclockwise current. In both cases, the interaction is governed by conservation of circulation $\Gamma$ around the island, which establishes a balance between the dissipation along the island in contact with the eddy and the dissipation along the island in contact with the generated current. Laboratory results and scaling analysis suggest that the interaction between an anticyclone (cyclone) and Okinawa Island should result in an instantaneous increase (decrease) of the Ryukyu Current transport and a delayed increase (decrease) of the Kuroshio transport. The estimated delays are in good agreement with those obtained with field measurements suggesting that the dynamics at play in the laboratory may be relevant for the flow around Okinawa Island.

Citation: Andres, M., and C. Cenedese (2013), Laboratory experiments and observations of cyclonic and anticyclonic eddies impinging on an island, J. Geophys. Res. Oceans, 118, 762-773, doi:10.1002/jgrc.20081.

\section{Introduction}

[2] Numerous eddies, both cyclonic and anticyclonic, impinge on the Ryukyu Island Chain in the western North Pacific where these islands separate the East China Sea from the Philippine Basin (Figure 1). The Kuroshio, the subtropical North Pacific's western boundary current, flows northeastward through the East China Sea and is shielded from these westward propagating eddies by the island chain, while the Ryukyu Current, which flows northeastward along the eastern side of the islands, is in the eddies' direct path. While the mean Ryukyu Current intensifies from about $5 \mathrm{~Sv}$ at the O-line off of Okinawa [Zhu et al., 2004] to $18 \mathrm{~Sv}$ off of Amami-oshima [Ichikawa et al., 2004], some eddy-induced transport variations are strong enough to reverse the direction of the flow by Okinawa [Zhu et al., 2004]. The transport through the O-line observed with ocean moorings ranges between -11 and $30 \mathrm{~Sv}$ (positive transport is directed northeastward).

[3] Transport variability for the Ryukyu Current crossing the O-line and the Kuroshio crossing the PN-line between 1993 and 2008 (Figure 2) (based on Andres et al. [2008a]) was determined from satellite altimetry calibrated with in

\footnotetext{
${ }^{1}$ Department of Physical Oceanography, Woods Hole Oceanographic Institution, Woods Hole, Massachusetts, USA.

Corresponding author: M. Andres, Department of Physical Oceanography, Woods Hole Oceanographic Institution, Woods Hole, MA 02543, USA. (mandres@whoi.edu)

(C) 2013 American Geophysical Union. All Rights Reserved. 2169-9275/13/10.1002/jgrc.20081
}

situ data from 2002 to 2004 in the Kuroshio [Andres et al., 2008a] and from 2000 to 2001 in the Ryukyu Current [Zhu et al., 2004]. These observations suggest that arrival of eddies at the Ryukyu Current east of Okinawa is also related with transport variability in the Kuroshio, though the magnitude of transport variability in the Kuroshio is not as pronounced as that in the Ryukyu Current (the reported standard deviations are 1.8 and $3.9 \mathrm{~Sv}$, respectively). The transport variability in the two currents is positively correlated at 60-day lag (Figure 2c). Positive transport anomalies in both currents are associated with the arrival of anticyclones along the eastern side of Okinawa, while negative transport anomalies are associated with the arrival of cyclones. The eddies themselves are generally not observed to pass through the gaps in the island chain and into the East China Sea. This suggests a "remote eddy effect" on the Kuroshio's transport, in contrast to the direct effect of eddies on the Ryukyu Current's transport.

[4] This study explores a possible mechanism by which eddies affect the Kuroshio transport inside the East China Sea, without propagating into that marginal sea. It is argued, based on laboratory results and observations, that the influence of eddies is transmitted to the East China Sea by boundary currents, herein called streamers, which are generated by the eddies and which flow around Okinawa. Laboratory experiments and field observations suggest that anticyclones and cyclones excite, respectively, a clockwise and counterclockwise flow around the island. This leads to transport variability on the western side of the island some time after an eddy impinges on the eastern side of the island. 

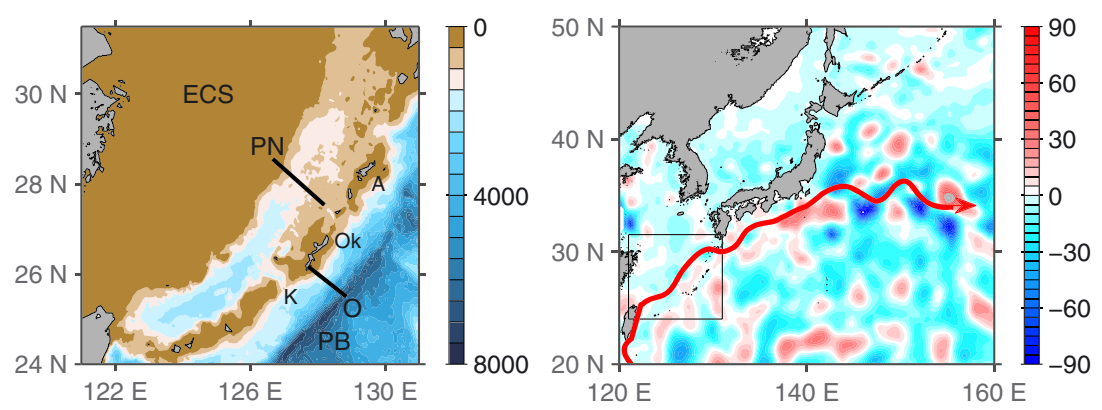

Figure 1. Maps of the western North Pacific. In the left panel the bathymetry (m) around the Ryukyu Islands is shaded. Amami-oshima (A), Okinawa (Ok), Kerama Gap (K), East China Sea (ECS), and Philippine Basin (PB) are labeled. Black lines labeled "PN" and "O" show the PN-line and O-line, respectively. Right panel shows a typical satellite sea surface height anomaly snapshot $(\mathrm{cm})$ contoured to highlight the ubiquitous westward-propagating eddies. Sea surface height anomaly data are from the AVISO mapped product. The Kuroshio path is shown (red curve). Black box indicates the area plotted in the left panel.

Conservation of circulation $\Gamma=\oint_{C} \mathbf{u} \cdot d \mathbf{s}$ around an island [Godfrey, 1989; Pedlosky et al., 1997] suggests that these streamers arise because dissipation generated where the eddy impinges on the island must be balanced by dissipation elsewhere along the island's perimeter. This mechanism is interpreted in the present study using laboratory experiments. Further, scaling arguments indicate that the dynamical balance controlling the streamer velocity around the island in the laboratory is not that used to describe a buoyant gravity current in which the velocity scales with the gravity wave speed. Rather, the streamer velocity is independent of the density contrast between the eddy and the ambient fluid.

[5] The interaction between a cyclonic eddy and a circular island/seamount and elliptical islands with small aspect ratio $(\sim 2)$ has been examined previously in the laboratory to determine under what conditions the arrival of a westward propagating barotropic cyclone leads to the generation of a second cyclone on the western side of the island [Cenedese, 2002; Adduce and Cenedese, 2004]. This work was extended to investigate the interactions of a cyclone with a chain of circular islands [Cenedese et al., 2005; Tanabe and Cenedese, 2008]. These studies found that the time-average flow around an island (averaged over the duration of the cyclone-island interaction) is governed by the requirement that circulation be conserved.

[6] Here, we further investigate eddy-island interactions for a long, narrow island with large aspect ratio $(\sim 7)$ to mimic the shape of Okinawa, and we focus on the time evolution of the interactions. Laboratory experiments are conducted for two classes of eddies: barotropic cyclones and baroclinic anticyclones. The experiments with barotropic cyclones are performed to verify that the conclusions from previous studies [Cenedese, 2002; Adduce and Cenedese, 2004] hold for this different island geometry. Furthermore, previous laboratory experiments on barotropic cyclones interacting with an island focused on the time-average (over the time of the interaction) circulation around the island. A novel aspect of the present study is that we consider not only the time-average circulation but also the evolving flow and circulation around the island, both for barotropic cyclonic and baroclinic anticyclonic eddy-island interactions. The experiments conducted with baroclinic anticyclones are novel, and the interaction of this class of eddies with an island has not been previously investigated in the laboratory.

[7] Both classes of eddies are relevant for the flow around Okinawa since both cyclones and anticyclones have been observed to impinge on this island [e.g., Zhu et al., 2008].

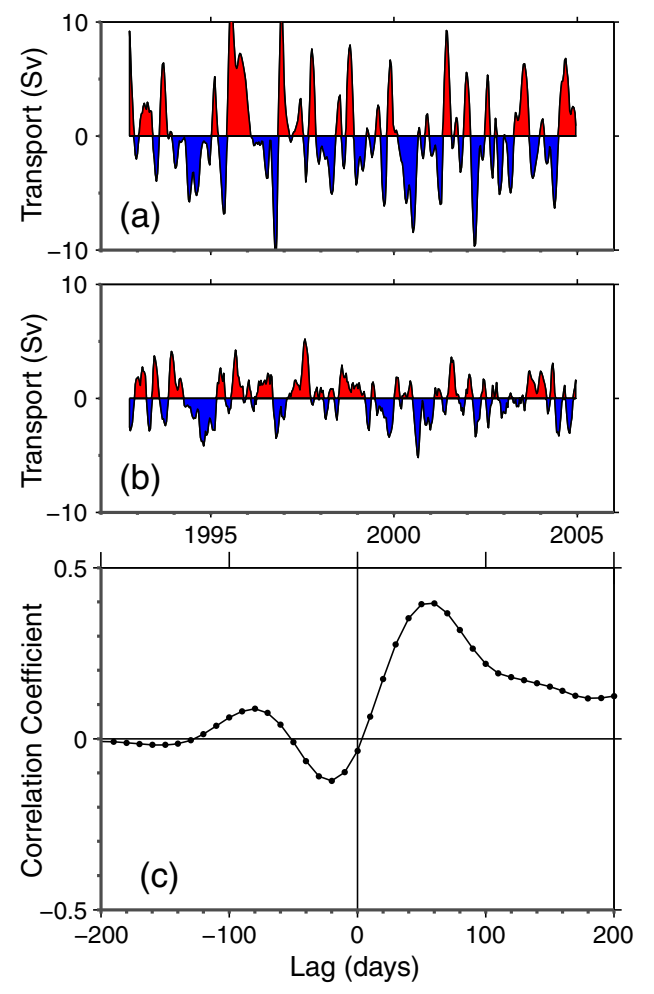

Figure 2. Transport anomalies determined from sea surface height anomaly, calibrated with in situ measurements (time series from Zhu et al. [2004] and Andres et al. [2008a]) for the Ryukyu Current crossing (a) the O-line and the Kuroshio crossing (b) the PN-line. Positive flow is towards the northeast; positive transport anomalies are shaded red; negative anomalies are shaded blue. (c) Lagged correlations between the transport anomalies for the total time series are shown. Correlation at positive (negative) lag indicates Kuroshio variability lagging (leading) Ryukyu Current variability. 
These eddies likely originate in the North Pacific interior due to baroclinic instability along the front between the westward-flowing North Equatorial Current and the shallow, eastward-flowing Subtropical Countercurrent [e.g., Qiu, 1999]. Since the isopycnals at this front slope upwards towards the north, cyclones (which can form along the southern side of the front by a meander trough that pinches off) are expected to carry water which is relatively uniform with depth while anticyclones (which can form along the northern side of the front by pinching off from a meander crest) hold the more stratified water (Figure 3). Since we expect that the cyclones arriving at Okinawa are more barotropic (less vertically sheared) than the anticyclones, we model these features in the laboratory with barotropic cyclones and baroclinic anticyclones, respectively.

[8] The paper is organized as follows. The experimental setup is described in section 2 and experimental results are presented in section 3. Theory and scaling analysis are presented in section 4. These results are then discussed in the context of field observations from Okinawa in section 5. Section 6 summarizes the results.

\section{Experimental Setup}

[9] Experiments are carried out in a glass tank of depth $60 \mathrm{~cm}$ and with a $60 \mathrm{~cm}$ square base (Figure 4). The tank is mounted on a rotating table with the axis of rotation aligned with the center of the tank. A plastic insert is placed in the tank to provide a bottom with slope, $\alpha=0.5$, where $\alpha=\tan \theta$ and $\theta$ is the angle between the insert and the horizontal plane. The sloping bottom provides a topographic $\beta$-effect, so the tank reproduces a $\beta$-plane approximation to a spherical earth, with the shallow side as the topographic equivalent to "north." Directions in the tank are defined with a right-hand coordinate system, $y$ is directed to the shallow side (north), $x$ is directed along the slope (east), and $z$ is vertically up. In the laboratory, a sloping bottom represents a topographic $\beta$-effect strictly only for unstratified fluids, e.g., the experiments with barotropic cyclones. For the experiments using baroclinic anticyclones, the slope provides an equivalent PV gradient in the lower layer, but there is not a direct representation of the PV gradient in the upper layer. However, the thermal wind coupling across the

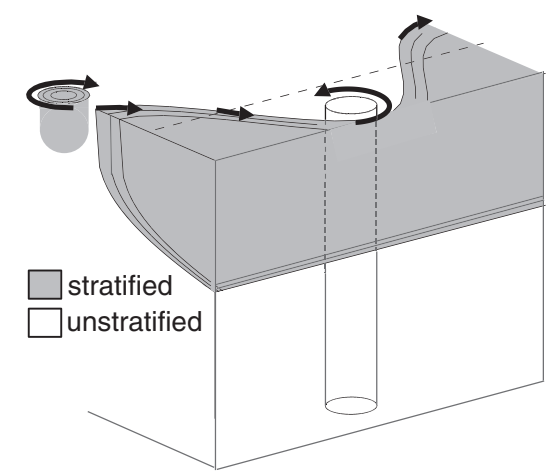

Figure 3. Schematic of a front between a stratified layer (gray) and a uniformly dense layer (white). Isopycnals (black lines) slope up towards the north and outcrop along the front. Meander crests about the mean path (dashed line) pinch off to form baroclinic anticyclones while troughs pinch off to generate barotropic cyclones.

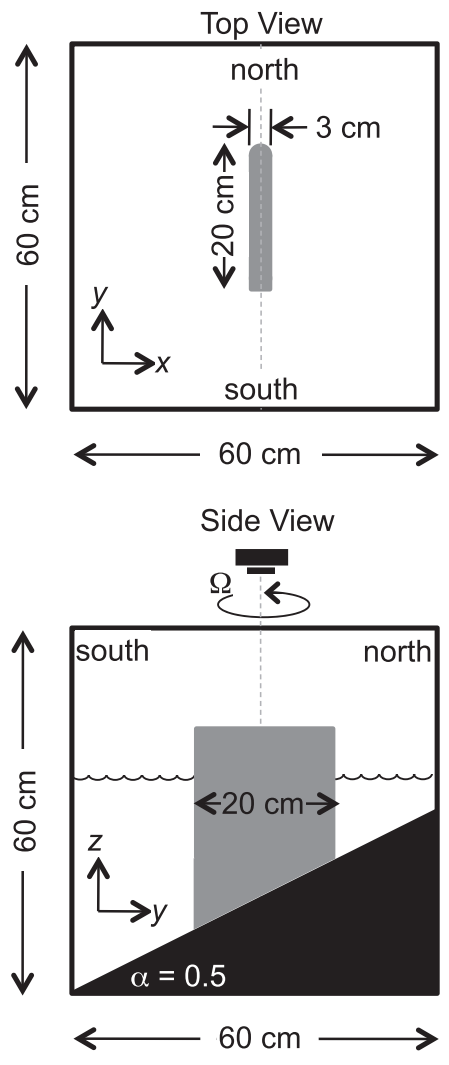

Figure 4. Experimental setup. Top view (upper panel) and side view (lower panel) showing the island (gray) and sloping bottom (black). Not to scale.

density interface ensures that the motion in the upper layer is also influenced by the lower-layer PV gradient. Hence, the essential features of a topographic $\beta$-effect are captured using a slope also in the experiment with baroclinic eddies.

[10] An island, $20 \mathrm{~cm}$ long and $3 \mathrm{~cm}$ wide with vertical sidewalls, is mounted flush with the bottom so its long axis is directed in the $y$-direction. One end of the island is semicircular and the radius of curvature is $1.5 \mathrm{~cm}$. For experiments with anticyclones, the curved end of the island is oriented towards the deeper side of the tank and for experiments with the cyclones, the curved end is towards the shallow side of the tank (Figure 5). We note here that this asymmetry in the island does not influence the experimental results. All the experiments terminate before the flow in the streamer reaches the square end of the island. Furthermore, some experiments were carried out with a symmetrical island (section 5) and the results are identical to those obtained with an asymmetric island (Figure 14). For experiments with anticyclones, water depth at the curved end is $\sim 10 \mathrm{~cm}$. For experiments with cyclones, it is $5 \mathrm{~cm}$. Table rotation rate $\Omega$ is set to give a Coriolis parameter $f$ between 0.75 and $1.5 \mathrm{~s}^{-1}$ (where $f=2 \Omega$ ). To record the evolution of an eddy's interaction with the island in the rotating reference frame, a camera is mounted on the top of the rotating table and is connected to a video recorder and a computer with imaging software.

[11] The tank is filled with filtered water of density $\rho_{T}$, equilibrated to room temperature, and degassed overnight. Densities are measured with a DMA58 Anton Paar densitometer with an accuracy of $10^{-5} \mathrm{~g} / \mathrm{cm}^{3}$. The water is 


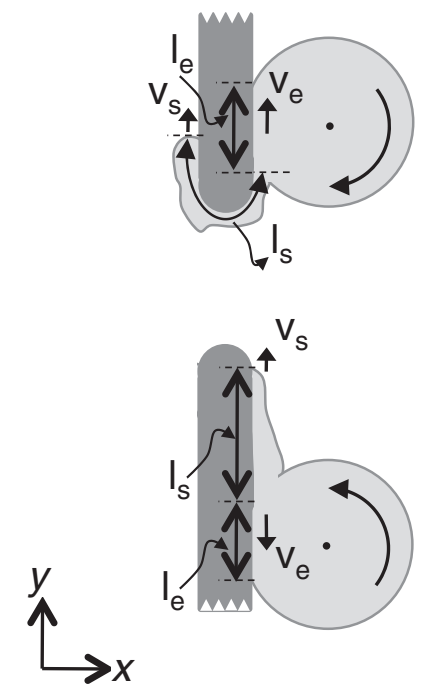

Figure 5. Eddy interaction with an island for an anticyclone (top) and for a cyclone (bottom). Eddy speed $\left(v_{e}\right)$, section length of the island in contact with the eddy $\left(l_{e}\right)$, streamer speed $\left(v_{s}\right)$, and streamer length $\left(l_{s}\right)$ are indicated. Not to scale.

allowed to spin up for $1 \mathrm{~h}$ before an eddy is generated in the tank. Eddies are generated as described below and buoyant paper pellets positioned on the free surface are used as tracers to determine the time-varying eddy speed $v_{e}(t)$ and streamer speed $v_{s}(t)$ by comparing video images separated by a time interval $d t$ (Figure 5). This interval varies for each velocity measurement but generally is between 2 and $10 \mathrm{~s}$. The eddy radius $R_{e}$ is defined as the radius where the eddy azimuthal velocity is maximum. The time-varying length of the streamer $l_{s}(t)$ is measured from individual video images, as is the section length $l_{e}(t)$ of the island in contact with the eddy (Figure 5). The eddy's evolution and the resulting flow around the island are recorded until the flow around the island spins down or the eddy moves away from the island.

[12] Experiments are designed to track the time-varying interaction of an eddy with the island for a range of eddy diameters $D_{e}=2 R_{e}$ and initial eddy velocities $v_{e}(0)$. Experimental parameters are listed in Table 1 for baroclinic anticyclones and Table 2 for barotropic cyclones.

\subsection{Baroclinic Anticyclones}

[13] Baroclinic anticyclones are generated as follows. The tank is filled with filtered seawater with density $\rho_{T}$ ranging between 1.02140 and $1.02261 \mathrm{~g} / \mathrm{cm}^{3}$ for different experiments. In a separate reservoir, filtered seawater is mixed with freshwater to produce a solution with density $\rho_{\text {res }}<\rho_{T}$, which is dyed with red food coloring to be visible during the experiment and in the video images. The dyed solution is pumped into the tank at $344 \mathrm{ml} / \mathrm{min}$ with an Ismatec BVP-Z pump through a tube with a $5 \mathrm{~mm}$ internal diameter and a foam diffuser at its end. This minimizes mixing so that the density of the eddy $\rho_{e} \approx \rho_{\text {res. }}$. The dyed reservoir water is

Table 1. Experimental Parameters for Baroclinic Anticyclones ${ }^{\mathrm{a}}$

\begin{tabular}{|c|c|c|c|c|c|c|c|c|c|c|c|c|c|}
\hline Experiment & $\underset{\left(s^{-1}\right)}{f}$ & $\begin{array}{c}\rho_{T} \\
\left(\mathrm{~g} / \mathrm{cm}^{3}\right)\end{array}$ & $\begin{array}{c}\rho_{e} \\
\left(\mathrm{~g} / \mathrm{cm}^{3}\right)\end{array}$ & $\begin{array}{c}g^{\prime} \\
\left(\mathrm{cm} / \mathrm{s}^{2}\right)\end{array}$ & $\begin{array}{c}\lambda \\
(\mathrm{cm})\end{array}$ & $\begin{array}{l}H_{T} \\
(\mathrm{~cm})\end{array}$ & $\begin{array}{l}H_{e} \\
(\mathrm{~cm})\end{array}$ & $\begin{array}{l}D_{e} \\
(\mathrm{~cm})\end{array}$ & $\begin{array}{c}\left. \pm \varepsilon D_{e}\right) \\
(\mathrm{cm})\end{array}$ & $\begin{array}{c}l_{e} \\
(\mathrm{~cm})\end{array}$ & $\begin{array}{l}\left. \pm \varepsilon l_{e}\right) \\
(\mathrm{cm})\end{array}$ & $\underset{(\mathrm{ml})}{Q}$ & Symbols \\
\hline 1 & 1.0 & 1.02231 & 1.02091 & 1.34 & 4.3 & 14 & 2 & 8.7 & 0.5 & 2.7 & 0.4 & 86 & Filled diamond \\
\hline 2 & 1.0 & 1.02237 & 1.02040 & 1.89 & 5.0 & 13 & 2 & 9.6 & 0.6 & 3.0 & 0.4 & 80 & Cross \\
\hline 3 & 1.0 & 1.02237 & 1.01615 & 5.97 & 8.8 & 13 & 1 & 13.7 & 0.6 & 4.0 & 0.4 & 149 & Filled square \\
\hline 4 & 1.0 & 1.02258 & 1.00866 & 13.35 & 13.7 & 14 & 1 & 18.0 & 0.7 & 4.3 & 0.4 & 154 & Filled triangle \\
\hline 5 & 1.0 & 1.02261 & 1.00624 & 15.70 & 14.8 & 14 & 1 & 20.7 & 0.8 & 4.3 & 0.4 & 107 & Filled circle \\
\hline 6 & 1.5 & 1.02144 & 1.02005 & 1.33 & 2.6 & 11 & 3 & 6.2 & 0.8 & 5.5 & 0.4 & 104 & Open diamond \\
\hline 7 & 1.5 & 1.02148 & 1.01620 & 5.07 & 5.0 & 11 & 2 & 7.0 & 0.8 & 5.5 & 0.4 & 77 & Open square \\
\hline 8 & 1.5 & 1.02165 & 1.01121 & 10.02 & 7.0 & 11 & 1 & 12.5 & 0.8 & 4.7 & 0.4 & 92 & Open triangle \\
\hline 9 & 1.5 & 1.02140 & 0.99850 & 21.99 & 10.4 & 11 & 1 & 15.6 & 0.8 & 6.2 & 0.8 & 132 & Open circle \\
\hline
\end{tabular}

${ }^{\mathrm{a}} \rho_{T}$ is the density of the ambient water in the tank, $\rho_{e}$ is the density of the buoyant eddy, $g^{\prime}$ is the reduced gravity given by equation (1), $\lambda$ is the baroclinic rossby radius of deformation calculated with equation $(2), H_{T}$ is the water depth where the eddy is introduced into the tank, $H_{e}$ is the approximate initial eddy depth, $D_{e}$ is the eddy diameter with the measurement uncertainty given by $\varepsilon\left(D_{e}\right), l_{e}$ is the distance over which the eddy interacts with the island with the measurement uncertainty given by $\varepsilon\left(l_{e}\right)$, and $Q$ is the initial volume of the eddy calculated from the pump flow rate and the time the pump is on. Symbols are used in Figures 7 and 11.

Table 2. Experimental Parameters for Barotropic Cyclones ${ }^{\mathrm{a}}$

\begin{tabular}{|c|c|c|c|c|c|c|c|c|c|c|c|c|c|c|}
\hline Experiment & $\begin{array}{c}f \\
\left(s^{-1}\right)\end{array}$ & $\begin{array}{c}\text { Ice } \\
\text { cubes }\end{array}$ & $\begin{array}{l}D_{e} \\
(\mathrm{~cm})\end{array}$ & $\begin{array}{l}H_{T} \\
(\mathrm{~cm})\end{array}$ & $\begin{array}{c}l_{s} \\
(\mathrm{~cm})\end{array}$ & $\begin{array}{c} \pm \varepsilon\left(l_{s}\right) \\
(\mathrm{cm})\end{array}$ & $\begin{array}{c}v_{s} \\
(\mathrm{~cm} / \mathrm{s})\end{array}$ & $\begin{array}{l} \pm \varepsilon\left(v_{s}\right) \\
(\mathrm{cm} / \mathrm{s})\end{array}$ & $\begin{array}{c}l_{e} \\
(\mathrm{~cm})\end{array}$ & $\begin{array}{c} \pm \varepsilon\left(l_{e}\right) \\
(\mathrm{cm})\end{array}$ & $\begin{array}{c}v_{e} \\
(\mathrm{~cm} / \mathrm{s})\end{array}$ & $\begin{array}{c} \pm \varepsilon\left(v_{e}\right) \\
(\mathrm{cm} / \mathrm{s})\end{array}$ & $\begin{array}{c}v_{s} l_{s} \\
\left(\mathrm{~cm}^{2} / \mathrm{s}\right)\end{array}$ & $\begin{array}{c}v_{e} l_{e} \\
\left(\mathrm{~cm}^{2} / \mathrm{s}\right)\end{array}$ \\
\hline 10 & 1.00 & 2 & 6.8 & 8.3 & 9.7 & 0.5 & 0.17 & 0.01 & 3.4 & 0.5 & 0.65 & 0.11 & 1.7 & 2.2 \\
\hline 11 & 1.00 & 1 & 5.1 & 12.4 & 8.2 & 0.5 & 0.17 & 0.01 & 2.6 & 0.5 & 0.51 & 0.11 & 1.4 & 1.3 \\
\hline 12 & 1.50 & 1 & 4.3 & 8.1 & 12.0 & 0.5 & 0.10 & 0.05 & 1.7 & 0.5 & 0.56 & 0.10 & 1.1 & 0.8 \\
\hline 13 & 1.50 & 2 & 4.3 & 8.5 & 9.7 & 0.5 & 0.29 & 0.02 & 3.0 & 0.5 & 0.68 & 0.14 & 2.8 & 2.0 \\
\hline 14 & 0.75 & 2 & 6.0 & 12.4 & 14.0 & 0.5 & 0.53 & 0.02 & 4.6 & 0.5 & 1.61 & 0.33 & 7.4 & 7.4 \\
\hline 15 & 0.75 & 1 & 5.1 & 10.2 & 11.9 & 0.5 & 0.30 & 0.01 & 4.0 & 0.5 & 0.90 & 0.15 & 3.6 & 3.6 \\
\hline
\end{tabular}

${ }^{\text {a }} D_{e}$ is the eddy diameter, $H_{T}$ is the water depth where the eddy impinges on the island, $l_{s}$ is the length of the streamer at time $t$ after the cyclone impinges on the island, with the measurement uncertainty given by $\varepsilon\left(l_{s}\right)$, and $v_{s}$ is an average velocity of the streamer given by $l_{s} / t$ with the measurement uncertainty given by $\varepsilon\left(v_{s}\right) . l_{e}$ is the distance over which the eddy interacts with the island during time interval $t$, with the measurement uncertainty given by $\varepsilon\left(l_{e}\right)$. $v_{e}$ is the azimuthal velocity of the eddy during this time, with the measurement uncertainty given by $\varepsilon\left(v_{e}\right)$. Fore experiment 12 only, the values are instantaneous values at $t=100 \mathrm{~s}$ obtained from the plots in Figure 10. 
delivered $\sim 1 \mathrm{~mm}$ below the free surface of the water in the tank. Experiments are run with $\rho_{e}$ between 0.99850 and $1.02005 \mathrm{~kg} / \mathrm{m}^{3}$. The associated reduced gravity $g^{\prime}$ ranges from 1.33 to $21.99 \mathrm{~cm} / \mathrm{s}^{2}$ (Table 1 ), where

$$
g^{\prime}=g\left(\rho_{T}-\rho_{e}\right) / \rho_{T}
$$

and $g=981 \mathrm{~cm} / \mathrm{s}^{2}$ is the acceleration due to gravity.

[14] The tube delivers buoyant water east of the island and $5 \mathrm{~cm}$ north of the island's southern tip (Figure 6) where the water depth $H_{T}$ ranges between 11 and $14 \mathrm{~cm}$ for different experiments. The buoyant water spreads radially outward until the Coriolis force, due to the rotation of the tank, alters the flow, and deflects it to the right (simulating the Northern Hemisphere, where $f>0$ ), thereby creating an anticyclonic eddy (clockwise flow). The interface between the ambient water and the dyed buoyant water deforms, and the maximum eddy thickness $H_{e}$ is at the center of the eddy in this 1 1 $1 / 2$-layer system (Figure 6). The flow rate of buoyant water producing the eddy is continuous for a time long enough to generate the eddy; this time depends on the baroclinic Rossby radius of deformation of the vortex

$$
\lambda=\sqrt{g^{\prime} H} / f
$$

where $H$ is a depth scale. Using $g^{\prime}$ and $f$ to control the eddy size, anticyclones with $D_{e}$ from 5.5 to $20.7 \mathrm{~cm}$ are generated for the various experiments. A total of nine experiments with baroclinic anticyclones are conducted comprising five with $f=1.0 \mathrm{~s}^{-1}$ and four with $f=1.5 \mathrm{~s}^{-1}$. For the nine experiments, the measured eddy radius $R_{e}$ is plotted as a function of $\lambda$ in Figure 7 , where $H_{T}$ is used as the depth scale in equation $(2) . H_{e}(t)$ is not measured explicitly, but visual estimates give a range of $H_{e}(0)$ between $\sim 1$ and $\sim 3 \mathrm{~cm}$, with the largest values occurring for the experiments with low $g^{\prime}$ and high $f$. As expected from equation (2), $R_{e}$ is larger when the density contrast between the eddy and the ambient water is larger and $R_{e}$ scales with $g^{\prime 1 / 2}$. In addition, $R_{e}$ is larger for a lower rotation rate and scales with $1 / f$.

[15] Although this method to generate baroclinic anticyclonic eddies is quite simple, we found that the eddy induces a relatively modest deflection of the interface between the
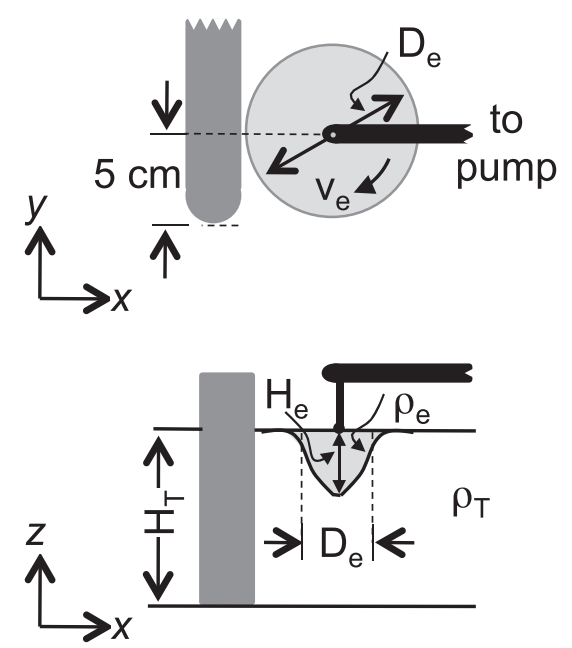

Figure 6. Schematic of the baroclinic anticyclone formation. Top view (upper panel) and side view (lower panel). Not to scale.

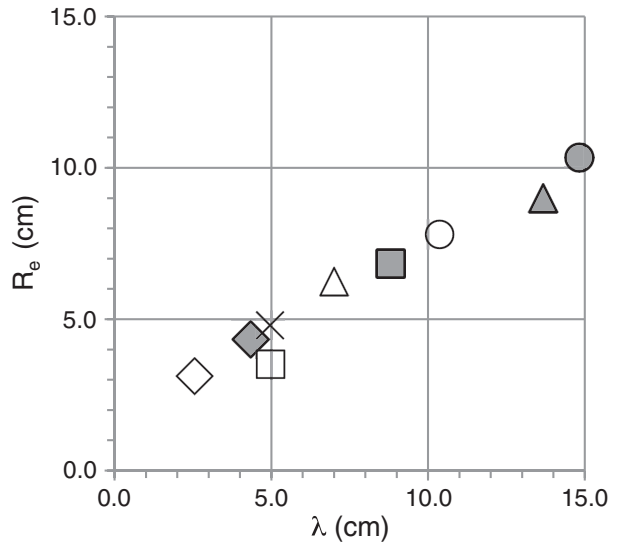

Figure 7. Measured eddy radius, $R_{e}$, versus the baroclinic Rossby radius of deformation, $\lambda$. Measurement errors in $R_{e}$ are approximately equal to symbol size. Open symbols are for experiments in which $f=1.5 \mathrm{~s}^{-1}$; others are for $f=1.0 \mathrm{~s}^{-1}$. See Table 1 for additional experimental parameters.

buoyant fluid and the ambient water. In order to conserve $\mathrm{PV}$, the column of ambient water below the buoyant eddy starts spinning anticyclonically but with a much weaker velocity than the buoyant eddy above. This weak bottom velocity leads to a weaker $\beta$-effect felt by the buoyant eddy; hence, the eddy only propagates westward slightly before it spins down. A similar behavior was observed in previous laboratory studies generating anticyclonic eddies with the same methodology [Cenedese and Linden, 1999; Cenedese et al., 2013]. To avoid premature eddy spin down, eddies are generated east of the island but at a distance slightly larger than $R_{e}$. During the experiment, care is taken to stop pumping the eddy buoyant fluid before the eddy's edge contacts the island, thus ensuring that the eddy is not externally forced during its interaction with the island. Furthermore, an eddy size of the order of the Rossby radius (equation 2) ensures that the eddy is baroclinically stable [Griffiths and Linden, 1981]. As suggested by previous studies [e.g., Cenedese, 2002], we assume that the westward speed of the eddy does not play a primary role in the dynamics regulating the interaction with the island.

\subsection{Barotropic Cyclones}

[16] Barotropic cyclones are generated according to the method of Whitehead et al. [1990], successfully used in previous studies [Cenedese, 2002, Adduce and Cenedese, 2004]. Once the tank filled with filtered fresh water is spun up, an ice cube is placed on the water surface east of the island and red food coloring is added over the ice to visualize the flow. As the cold melt-water sinks, it generates a dense plume, which entrains the surrounding water. This entrained water flows radially inward towards the sinking plume and is deflected to the right (in the northern hemisphere) by the Coriolis force, thereby generating a cyclone (counterclockwise flow) throughout the water column (i.e., $H_{e}=H_{T}$ ). For the cyclones, $D_{e}$ is controlled by the size of the ice cube. Cyclones interact directly with the bottom and move westward due to the topographic $\beta$-plane and typically can propagate across the entire tank without spinning down significantly (Figure 10b).

[17] Experiments are conducted for a range of $D_{e}$ between 4.3 and $6.8 \mathrm{~cm}$ and for three different values of $f 0.75,1.0$, 
and $1.5 \mathrm{~s}^{-1}$ (Table 2). The exact location where the ice cube is introduced into the tank is difficult to control accurately. Hence, the water depth where the cyclones are generated varies between experiments, though the water depth at the northern tip of the island is always $5 \mathrm{~cm}$. The water depth where the cyclones first impinge on the island $H_{T}$ is measured and ranges between 8.1 and $12.4 \mathrm{~cm}$ (Table 2).

\section{Experimental Results}

[18] The interaction of an eddy with an island results in the generation of a streamer that detaches from the eddy and flows along the island's perimeter. Anticyclones generate streamers that flow clockwise around the island (Figure 5, top), while cyclones generate streamers that flow counterclockwise around the island (Figure 5, bottom). For both classes of eddies, the streamer continues to flow as long as the eddy is in contact with the island and there is eddy motion $\left(v_{e}\right)$ tangent to the island. For anticyclones, the eddy motion tangent to the island ceases because the eddy itself spins down. For cyclones, the eddy often continues to spin throughout the experiment, but it eventually propagates past the island and the streamer flow ceases once the cyclone is no longer in contact with the island. In addition to the opposite sense of propagation of the streamer flow, there are other qualitative differences between the interactions of anticyclones and cyclones with islands as described below.

\subsection{Baroclinic Anticyclones}

[19] When a baroclinic anticyclonic eddy impinges against the island's eastern side over a distance $l_{e}$ (Figure 5, top and Figures $8 \mathrm{a}$ and $8 \mathrm{~b}$ ), buoyant fluid detaches from the eddy and forms a streamer emanating from the eddy's southwestern edge (i.e., just south of the region where the eddy flow along the island is northward). This streamer progresses anticyclonically (clockwise) around the island's perimeter over a distance $l_{s}$.

[20] The time evolution of an anticyclonic eddy interacting with an island and the resulting streamer is illustrated for two representative experiments in Figure 9. As the eddy continues to interact with the island, the location of its center remains fixed relative to the island (as shown in Figures 8a and 8b), but $v_{e}$ decreases as the eddy spins down (Figure 9, crosses). In addition, $H_{e}$ decreases as the tilted density interface between the layers relaxes. Meanwhile, the streamer progresses around the island ( $l_{s}$ increases, Figure 9 , triangles) with a velocity, $v_{s}$, that decreases in time (Figure 9, squares). If the anticyclonic eddy were free to evolve without interacting with the island and generating a streamer, the eddy spin down would cause the azimuthal velocity to decrease in time with a consequent flattening of the interfacial slope. Provided that the eddy conserves mass (i.e., there is no filamentation or baroclinic instability), this flattening would result in an increase of the eddy diameter $D_{e}$ as the eddy depth $H_{e}$ decreased [Ou and Gordon, 1986; Hedstrom and Armi, 1988]. However, in the present experiments, $D_{e}$ and $l_{e}$ are observed to remain fairly constant during the eddy's interaction with the island (Figures $8 \mathrm{a}$ and $8 b)$. This behavior suggests that the streamer is siphoning away just enough buoyant water for the eddy diameter to remain constant. For the parameter range investigated here, the eddy and the streamer spin down completely before the streamer reaches the northwestern edge of the island.
[21] It is important to notice that the dynamical balance that regulates the streamer generation and development is different than that of a buoyant gravity current near a vertical wall. For the latter, the condition of no-flow normal to a vertical wall implies that the Coriolis force parallel to the wall is zero and buoyant water flows parallel to the island's wall with the Coriolis force directed normal to the wall balanced by the pressure gradient due to the density difference between the buoyant fluid and the ambient water. The resulting buoyant "coastal" current hugs the wall on its right looking downstream (in the northern hemisphere). This dynamical balance implies that the velocity of the buoyant gravity current should scale with the gravity wave speed $\sqrt{g^{\prime} h}$ where $h$ is the buoyant current depth [Lentz and Helfrich, 2002; Simpson, 1997]. Although the value of $h$ is not measured, visual estimates give a range between $\sim 1$

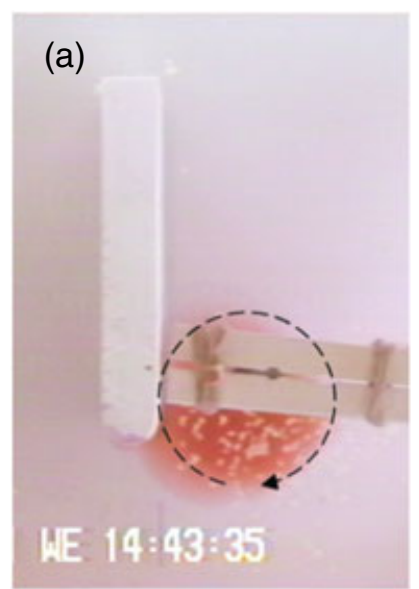

(c)

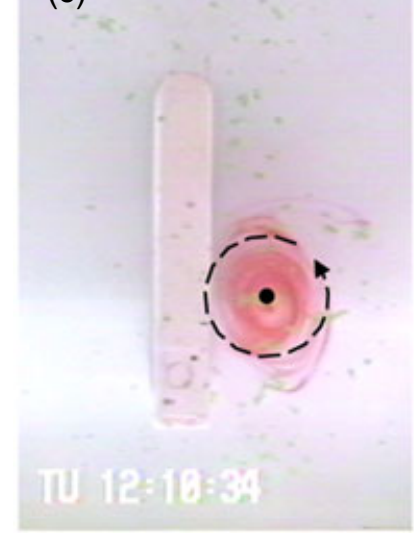

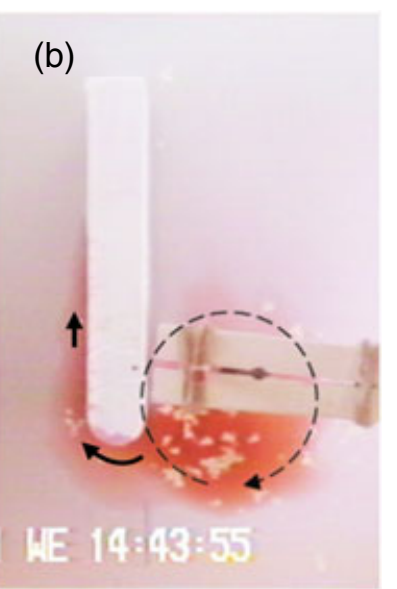

(d)

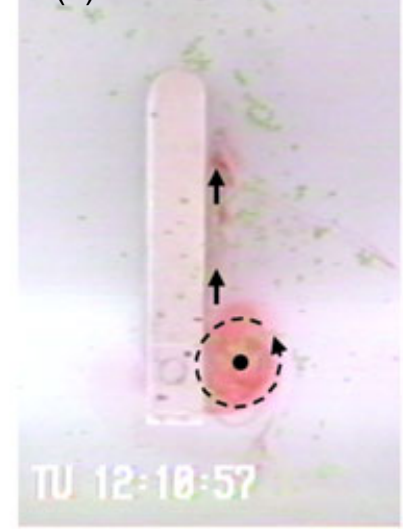

Figure 8. Top shows top views of a baroclinic anticyclone (a) $2 \mathrm{~s}$ and (b) $22 \mathrm{~s}$ after impinging on the island. Dashed circle indicates $D_{e}(6 \mathrm{~cm})$, which is approximately constant throughout the experiment while $v_{e}$ decreases. In Figure $8 \mathrm{a}$, the streamer is rounding the southern tip of the island and in Figure $8 \mathrm{~b}$, it is clearly visible along the island's western edge (part of the streamer's view is obstructed by the island). In this example (not listed in Table 1 or included in Figure 7), $g^{\prime}=2.0 \mathrm{~g} / \mathrm{cm}^{3}$ and $f=1.5 \mathrm{~s}^{-1}$. Bottom shows top views of a cyclone (c) $1 \mathrm{~s}$ and (d) $24 \mathrm{~s}$ after impinging on the island. During this time, the eddy (indicated with the dashed circle) diameter decreases by about $2 \mathrm{~cm}$. Concurrently, the eddy center moves southward by $\sim 3.5 \mathrm{~cm}$. In this example (experiment 19 in Table 2), $f=0.75 \mathrm{~s}^{-1}$. 

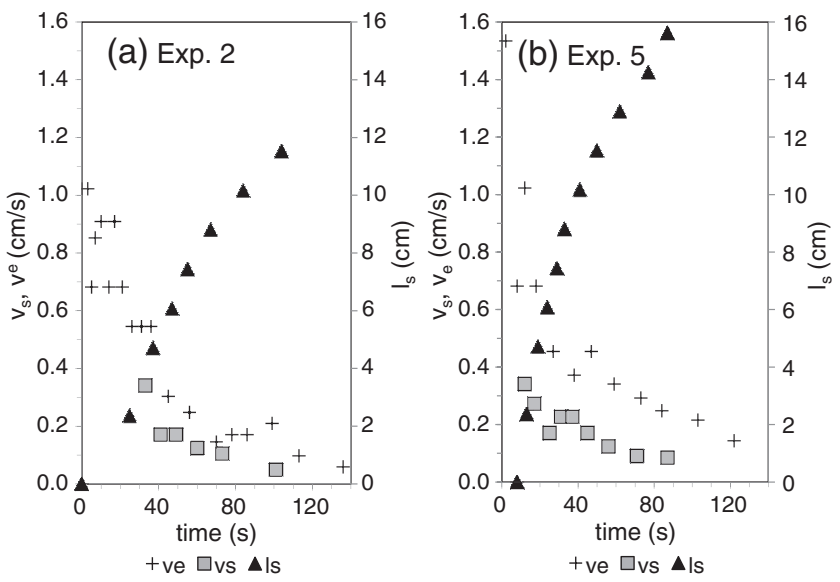

Figure 9. Time evolution of the streamer velocity, eddy azimuthal velocity, and length of the streamer for a baroclinic anticyclone impinging on an island. (a) Experiment 2 in which $l_{e} \sim 3.0 \mathrm{~cm}$ and (b) experiment 5 in which $l_{e} \sim 4.3 \mathrm{~cm}$. Other experimental parameters are listed in Table 1.

and $\sim 2 \mathrm{~cm}$. Hence, if the leading order dynamical balance were the one described above, one would expect the streamer velocity to be different between experiments 2 (Figure 9a) and 5 (Figure 9b) because they have very different $g^{\prime}$ values. In particular, $g^{\prime}$ of experiment 5 is approximately eight times larger than that of experiment 2 , which should give rise to a streamer that is 2.8 times faster than the streamer in experiment 2. Furthermore, the magnitude of the gravity wave speed $\sqrt{g^{\prime} h}$ is $\sim 1.5 \mathrm{~cm} / \mathrm{s}$ for experiment 2 and $\sim 4.5 \mathrm{~cm} / \mathrm{s}$ for experiment 5 . However, Figure 9 clearly suggests that the streamer velocities in these two experiments are approximately equal and much smaller than $\sqrt{g^{\prime} h}$, which suggests that the dynamics regulating the generation and motion of the streamers are different than the dynamics regulating buoyant gravity currents near a wall.

\subsection{Barotropic Cyclones}

[22] The arrival of a self-propagating barotropic cyclone on the island's eastern side causes southward flow along the island over a distance $l_{e}$ accompanied by a streamer, which begins at the eddy's northwestern edge and flows cyclonically (counterclockwise) around the island's perimeter over a distance $l_{s}$ (Figure 5, bottom). The streamer flows along the island until it reaches the northern tip of the island. Thereafter, the streamer's leading edge continues westward and looses contact with the island's perimeter (not shown) and at this point, $l_{s}$ is the distance between the eddy's northwestern edge and the island's northernmost tip.

[23] In contrast to the experiments with anticyclones, whose center remains fixed relative to the island, the cyclone propagates southward along the island (Figures $8 \mathrm{c}$ and $8 \mathrm{~d}$ ). The behavior of a single eddy near a wall can be found by superimposing two eddies of equal and opposite strength, the so-called "image-effect" [Kundu and Cohen, 1990]. The cyclonic eddy and its image, on the other side of the wall, couple to form a dipole which propagates southward (the image-effect should be at work also for baroclinic anticyclones and cause them to propagate northward. However, the anticyclonic eddies are not observed to move along the island, possibly because they are weaker than the barotropic cyclones and spin down rapidly). The cyclone's motion causes the streamer to lengthen on both ends as the streamer's leading edge progresses away from the eddy and as the eddy feeds the streamer's trailing edge. Once the cyclone reaches the southern tip of the island, it continues to self-propagate westward (steered by the bottom topography), looses contact with the island, and $v_{e}=0$ on the island even though the eddy itself has not spun down. At this point, the streamer also stops moving and $v_{s}=0$. In addition, the cyclone's diameter decreases during the interaction as the streamer siphons off a large amount of eddy fluid (compare Figures $8 \mathrm{c}$ and $8 \mathrm{~d})$. Thus, $l_{e}$ decreases during the interaction in contrast to the anticyclone experiments where $l_{e}$ remains nearly constant during an experiment.

[24] Figure 10 shows an example of the time evolution of a barotropic cyclone interacting with an island and its streamer. For most of the experiments with barotropic cyclones, the eddy impinges on the island fairly close to its southern tip. Hence, the duration of the interaction is quite short because the eddy moves south and then westward past the island, and detailed measurements like those shown in Figure 10 are not attainable. However, the cyclonic eddy in experiment 12 impinged on the island fairly close to the northern tip and the long interaction with the island allowed meaningful measurements to be taken. For this experiment, $D_{e}$ and $l_{e}$ decrease by a factor of 3 during the $170 \mathrm{~s}$ interaction (Figure 10a). In addition, the location of the eddy's center when the eddy hits the island is about $5 \mathrm{~cm}$ south of the northern tip of the island and during the interaction, it moves $15 \mathrm{~cm}$ southward. During this interaction, $v_{e}$ remains nearly constant $(\sim 0.5 \mathrm{~cm} / \mathrm{s})$, while the streamer, whose length increases as the eddy moves southward, slows from $v_{s}=0.17$ to $\sim 0.05 \mathrm{~cm} / \mathrm{s}$ (Figure 10b).

\section{Scaling and Discussion}

[25] The laboratory results can be interpreted by considering the horizontal momentum equation

$$
\frac{\partial \mathbf{u}}{\partial \mathrm{t}}+(\zeta+f) \hat{\mathbf{k}} \times \mathbf{u}=-\nabla\left(\frac{p}{\rho}+\frac{|\mathbf{u}|^{2}}{2}\right)+\operatorname{Diss}(\mathbf{u}) .
$$

[26] Here, $\mathbf{u}$ is the horizontal velocity vector, $\zeta$ is the vertical component of relative vorticity, $\hat{\mathbf{k}}$ is the unit vector in the vertical, $p$ is pressure, and $\operatorname{Diss}(\mathbf{u})$ is the dissipation due to friction. Following the arguments of Cenedese [2002], Pedlosky et al. [1997], and Godfrey [1989], equation (3) can be integrated along the island's boundary $C$ to calculate the circulation around the island. After this integration, the second term on the LHS is zero because there is no flow normal to the island and the first term on the RHS is zero because it is a perfect differential. This leaves

$$
\oint_{C} \frac{\partial u_{\tan }}{\partial t} d s=\oint_{C} D i s s\left(u_{\tan }\right) d s .
$$

[27] The term on the LHS is zero because the tangential component of velocity $u_{\text {tan }}$ is zero over the island's boundary when using a no-slip boundary condition. If friction is due to lateral dissipation (rather than bottom friction), 

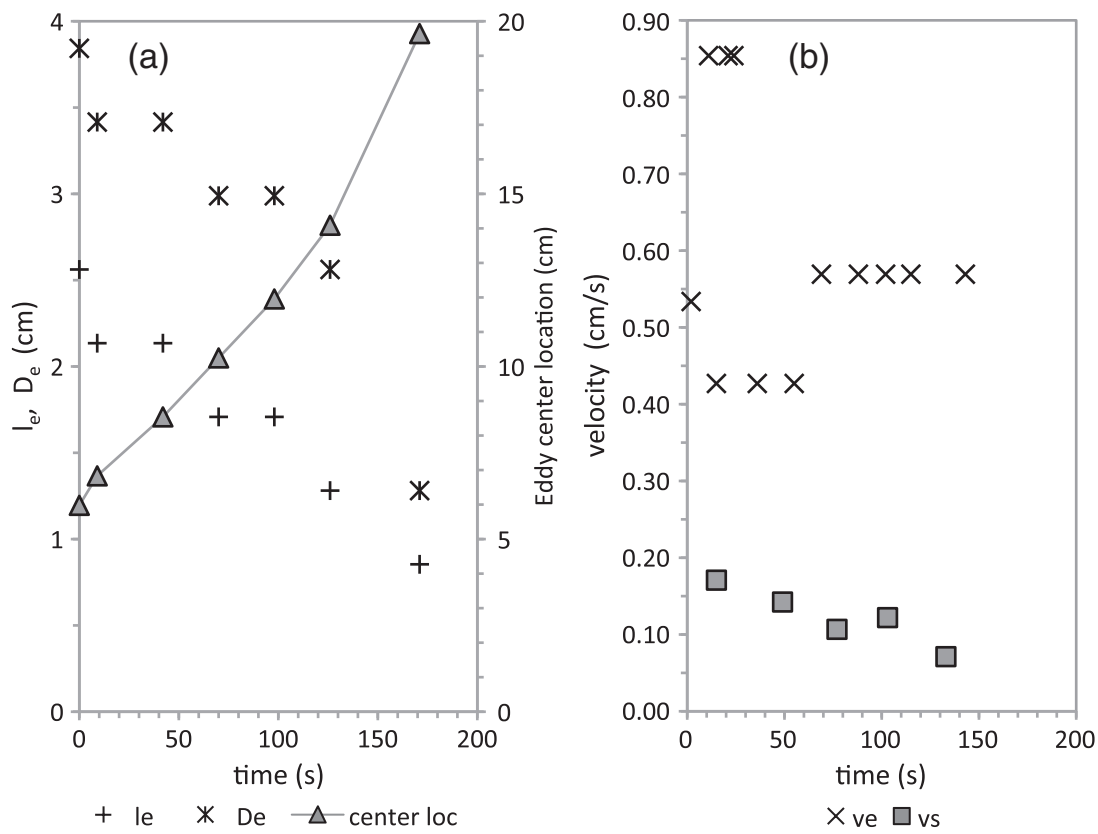

Figure 10. Time evolution for a barotropic cyclone impinging on an island (experiment 12). (a) The decrease in eddy size $\left(D_{e}\right.$ and $\left.l_{e}\right)$ and the change in location of the eddy center relative to the northern tip of the island are shown. (b) The velocities of the eddy and streamer are shown.

then $\operatorname{Diss}\left(u_{\tan }\right)=\nu \nabla^{2} u_{\tan }$ and although $u_{\tan }$ is zero on the island's boundary, $\nabla^{2} u_{\tan } \neq 0$. Hence, equation (4) reduces to

$$
\oint_{C} \nu \nabla^{2} u_{\tan } d s=0
$$

[28] With a no-slip boundary, the streamer's tangential velocity changes from zero on the island's boundary to $v_{s}$ across a thin boundary layer whose thickness is $\delta$. The velocity difference across the boundary layer is $v_{s}-0=v_{s}$ and $\nu \nabla^{2} u_{\tan }$ scales as $\nu\left(v_{s} / \delta^{2}\right)$. Likewise, the velocity difference across the boundary layer between the eddy and the island is $v_{e}$ and $\nu \nabla^{2}$ $u_{\tan }$ scales as $\nu\left(v_{e} / \delta^{2}\right)$. Using these in equation (5) and treating $\delta$ and $\nu$ as constants gives

$$
v_{s} l_{s}=-v_{e} l_{e}
$$

where $v_{e}$ and $v_{s}$ are defined positive for cyclonic flow around the island and negative for anticyclonic flow. According to equation (6), since the initial circulation around the island is zero, the circulation where the eddy interacts with the island $\left(v_{e} l_{e}\right)$ must be balanced by a circulation of the same magnitude and opposite direction elsewhere along the island. This happens in the streamer where $v_{s} l_{s}$ balances $v_{e} l_{e}$. Equation (6) holds both for the instantaneous and for the time-average circulation around the island.

[29] Experimental results for baroclinic anticyclones impinging on the island are consistent with the scaling in equation (6). For each of the nine experiments, values of $v_{s} l_{s}$ and $v_{e} l_{e}$ are plotted in Figure 11. This figure shows both the time-average values (large symbols) and the instantaneous values (small symbols) of the circulation around the island along the eddy $\left(-v_{e} l_{e}\right)$ and the streamer $\left(v_{s} l_{s}\right)$. For a given eddy-streamer pair, the circulation magnitudes $\left(\left|v_{s} l_{s}\right|\right.$ and $\left.\left|v_{e} l_{e}\right|\right)$ around the island decrease with time as the system spins down. In general, experiments conducted with larger $f$ have greater $\left|v_{s} l_{s}\right|$ and $\left|v_{e} l_{e}\right|$ as can be seen by the results for $f=1.5 \mathrm{~s}^{-1}$ (open symbols) which cluster towards the upper right-hand side of the domain in Figure 11 relative to the results for $f=1.0 \mathrm{~s}^{-1}$ (solid symbols).

[30] Albeit some scatter in the results, particularly for the instantaneous values, in general, $v_{s} l_{s}$ balances $v_{e} l_{e}$ for each eddy-streamer pair, both for the averaged values and throughout the duration of the interaction. Perfect agreement with equation (6) would give results that fall along the

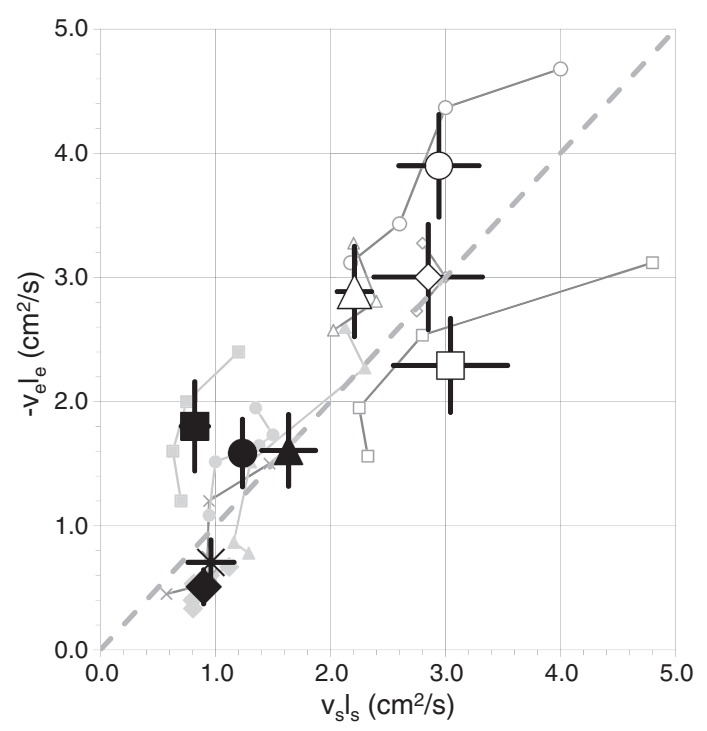

Figure 11. Baroclinic anticyclone experiments. Timeaverage (large symbols) and time-evolving (small symbols) values of the circulations $-v_{e} l_{e}$ and $v_{s} l_{s}$. Dashed gray line shows 1:1. Symbols as in Figure 4 and Table 1. Error bars for the time-average values are calculated by error propagating the measurement uncertainties listed in Table 1. 
dashed line in Figure 11; within the experimental errors, the time-average results do cluster along this line suggesting that the balance described in equation (6) holds for these experiments.

[31] Experimental results for barotropic cyclones impinging on an island are also consistent with the scaling in equation (6). Figure 12 shows averaged values of $v_{s} l_{s}, v_{e} l_{e}$ for each experiment (Table 2) measured over a long fraction of the duration of the eddy-island interaction. The time evolution of a cyclone-streamer pair is shown in Figure 10 for an experiment in which the interaction was long and detailed measurements could be obtained.

[32] These results are consistent with previous results for barotropic cyclonic eddies interacting with a circular island [Cenedese, 2002] and confirm that the scaling in equation (6) holds both for circular islands and for the elongated island investigated in the present study. Figure 12 shows the results from the previous experiments, which were conducted with self-propagating vortexes (black dots) and with vortexes advected in a background flow (black crosses).

\section{Comparison With Field Observations}

[33] The cyclones and anticyclones which arrive by Okinawa with approximately 100 -day period have azimuthal velocities $O$ $(10 \mathrm{~km} /$ day) and their diameter is $O(100 \mathrm{~km})$ [e.g., Konda et al., 2005; Zhu et al., 2008; Andres et al., 2008a]. While the laboratory experiments were conducted for an island whose long axis was oriented along the meridional direction, Okinawa Island is oriented at an angle of about $45^{\circ}$ with the meridional direction (Figure 13). The experimental procedure is repeated for both a baroclinic anticyclone and a barotropic cyclone impinging on a tilted island, oriented at an angle of approximately $45^{\circ}$ with the isobaths, to qualitatively compare the laboratory results with satellite and in situ observations around Okinawa Island (Figure 14). Indeed, the streamer induced by a baroclinic anticyclonic eddy continues to flow clockwise around this tilted island until the anticyclone has spun down (left panel). The streamer induced by the barotropic cyclone flows counterclockwise to the island's northwestern edge, where the streamer detaches from the island and flows westward, constrained by the topographic $\beta$-plane to follow isobaths (right panel). Hence, based on the laboratory experiments, we expect the interaction between an anticyclone (cyclone) and Okinawa Island to result in an instantaneous increase (decrease) of the Ryukyu Current transport and a delayed increase (decrease) of the Kuroshio transport (Figure 13). This is consistent with the field observations, which show a lagged positive correlation between the two current transport anomalies (Figure 2).

[34] In addition to this qualitative evidence from the laboratory experiments suggesting that anticyclonic and cyclonic eddies induce clockwise and counterclockwise streamers, respectively, when they impinge on Okinawa, field observations are consistent with the scaling discussed in section 4 and suggest that this mechanism may be responsible for the observed co-variability between the Kuroshio and Ryukyu Current transport near Okinawa. The total distance along the $500 \mathrm{~m}$ isobath around Okinawa is about $500 \mathrm{~km}$ (Figure 13). The $\mathrm{O}$-line and PN-line are separated by roughly $300 \mathrm{~km}$ in the clockwise direction and $200 \mathrm{~km}$ in the counterclockwise direction. Since cyclones and anticyclones in the western North Pacific have approximately similar size and angular velocity, the laboratory results discussed above suggest, to first order, that the shorter route (i.e., the counterclockwise route taken

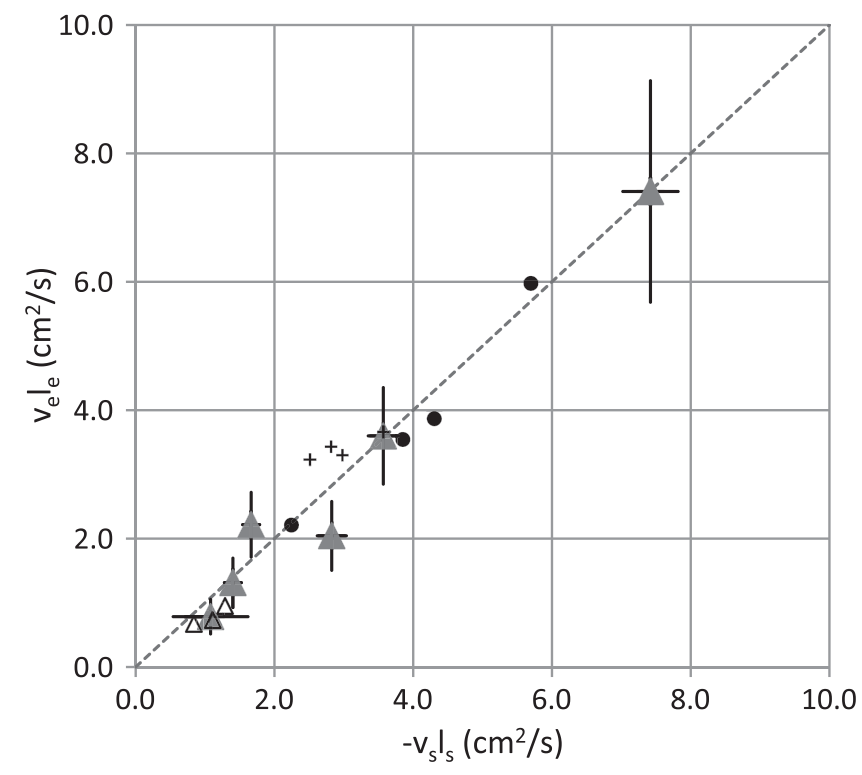

Figure 12. Barotropic cyclone experiments. Present experiments are shown with triangles (experimental parameters listed Table 2). Values are calculated over a long fraction of each experiment's interaction time and hence represent averages for each experiment, except for the open triangles, which are instantaneous values from experiment 12 (Figure 10) with the solid triangle showing the average of these three instantaneous values. Errors bars are calculated by error propagating the measurement uncertainties listed in Table 2. Results from previous experiments are shown for comparison [see Cenedese, 2002, Table 1]. These were conducted with self-propagating vortexes (black dots) and with vortexes advected in a background flow (black crosses). Dashed gray line shows 1:1. 

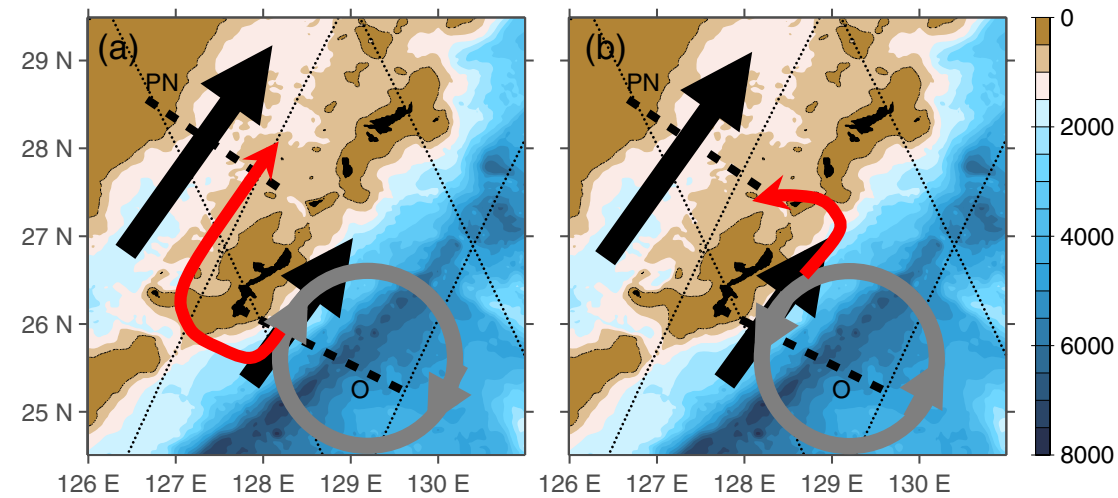

Figure 13. Schematic of the circulation induced around Okinawa Island by (a) an anticyclone and (b) a cyclone. Depth is shaded (m). Heavy dashed lines indicate PN-line (PN) and O-line (O) with black arrows indicating the mean Kuroshio and Ryukyu Current crossing these lines, respectively. Red arrows indicate the streamers generated by the eddies (gray circles) impinging on Okinawa. Thin dashed lines indicate satellite tracks.
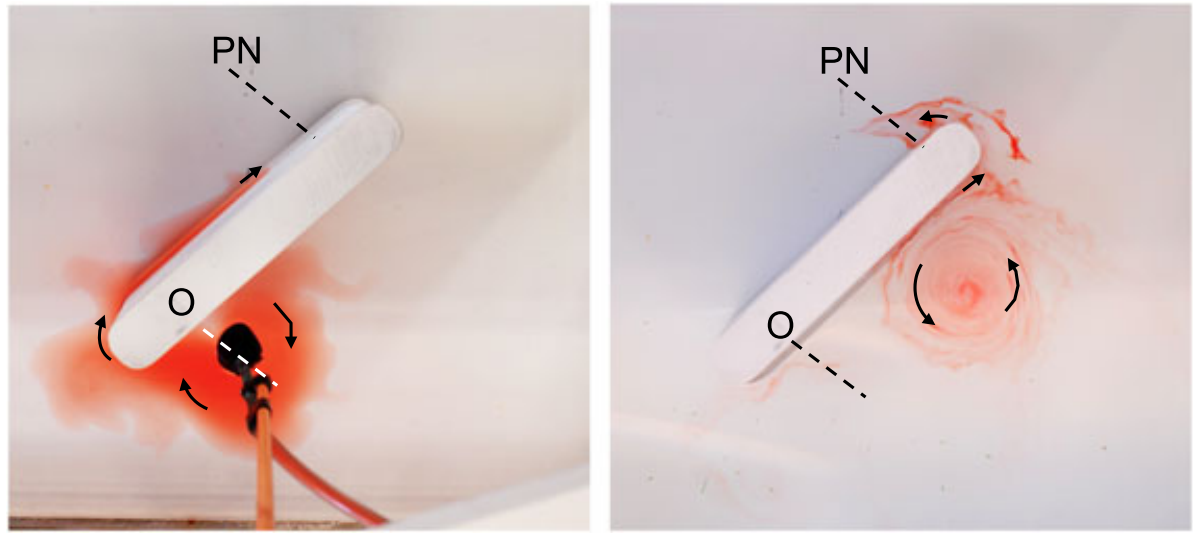

Figure 14. Top views of laboratory experiments for a baroclinic anticyclone (left panel) and a barotropic cyclone (right panel) impinging on a tilted island. Arrows indicate direction of flow. Dashed lines represent the O-line and PN-line as in Figure 12.

by the cyclones' streamers, Figure 13b) should result in a shorter delay between the Ryukyu Current and Kuroshio transport anomalies than the delay due to streamers flowing along the longer route (i.e., the clockwise route taken by the anticyclones' streamers, Figure 13a). From the scaling in equation (6) and considering that $v_{e} \sim 10 \mathrm{~km} /$ day is a typical eddy azimuthal speed, $l_{e} \sim 100 \mathrm{~km}$ is a typical eddy size, and $l_{s} \sim 200 \mathrm{~km}$, we obtain a mean streamer velocity $v_{s} \sim 5 \mathrm{~km} /$ day. This is the predicted velocity of the streamer flowing clockwise around Okinawa. When considering the counterclockwise distance of $l_{s} \sim 300 \mathrm{~km}$, we obtain a mean streamer velocity $v_{s} \sim 3.3 \mathrm{~km} /$ day. Hence, the delay between the arrival of a cyclonic eddy at the O-line (where the southeastward eddy azimuthal velocity opposes the northeastward mean flow and causes a Ryukyu Current transport decrease, Figure 13b) and a transport decrease along the PN-line (where the counterclockwise-flowing streamer opposes the mean northeastward Kuroshio transport, Figure 13b) can be estimated to be approximately $200 \mathrm{~km} / 5 \mathrm{~km} /$ day $=40$ days. In contrast, an anticyclone's streamer travels $300 \mathrm{~km}$ clockwise between the two lines at $\sim 3.3 \mathrm{~km} /$ day, leading to a predicted lag of 90 days. In this case, the arrival of an anticyclone at the O-line leads to northeastward eddy azimuthal velocity that increases the
Ryukyu Current transport and the clockwise streamer flow adds to the northeastward flowing Kuroshio transport, also leading to a transport increase (Figure 13a). Hence, the laboratory experiments predict that negative transport anomalies (decreases in transport) in the Ryukyu Current and Kuroshio are positively correlated at 40-day lag, while positive transport anomalies are positively correlated at 90-day lag (Figure 15, dashed and dotted lines, respectively).

[35] Next, we consider observations from the O-line and PN-line in more detail to evaluate whether the dynamics and scaling deduced from the laboratory experiments are relevant for the observations. The in situ measurements and the use of satellite altimetry to infer transports are described in detail in Andres et al. [2008a, 2008b] for the PN-line and Zhu et al. [2003, 2004] for the O-line. Time series of transports in the Kuroshio and Ryukyu Current were measured in separate experiments with high temporal and spatial resolution using combinations of moored ADCPs, current sensors, and pressure-sensor-equipped inverted echo sounders. In each region, these time series from in situ measurements were then used to determine empirical relationships between the surface geostrophic velocities, determined from satellite-measured sea surface height gradients across the currents (from along-track 


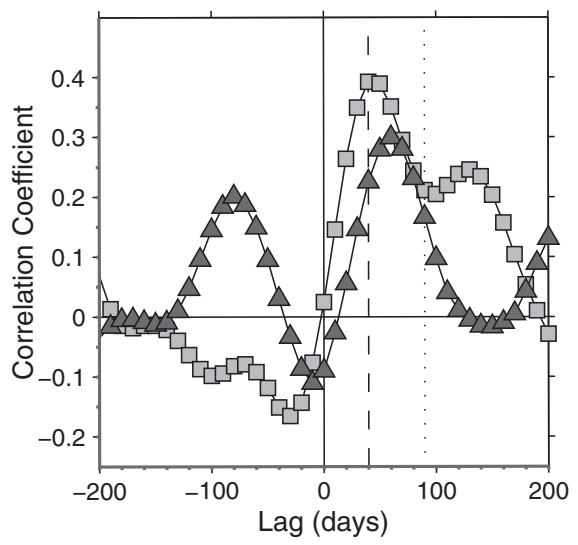

Figure 15. Lagged correlations between Ryukyu Current and Kuroshio positive transport anomalies (triangles) and negative transport anomalies (squares). Lines show lags predicted by the scaling discussed in section 4 for positive (dotted line) and negative (dashed line) transport anomalies. Correlation at positive (negative) lag indicates Kuroshio variability lagging (leading) Ryukyu Current variability.

satellite data) and the total (surface-to-bottom) transports. As a result, time series for the Kuroshio and Ryukyu Current, each essentially 40-day low pass filtered, are available since the beginning of the satellite altimetry record in late 1992.

[36] Both currents' transports (determined in the manner described above) exhibit significant transport variability around $100-200$ day period. Further, the 100-200 day variability is coherent between the two currents, with 60-day lag (Figure 2c). Analysis of mapped satellite altimetry (available at 7-day interval) from the region shows that variability in Ryukyu Current transport corresponds to the arrival of westward propagating cyclones and anticyclones from the ocean interior as their onshore side impinges on the Ryukyu Current [Andres et al., 2008a]. It is not clear from the mapped altimetry exactly how these signals propagate into the East China Sea (the spatial resolution of satellite data is too coarse for this), but it was noted by Andres et al. [2008a] that the arrival of eddies at the O-line leads Kuroshio transport variability at the PN-line. While the two currents are well correlated at 60-day lag, no physical mechanism linking Kuroshio transport variability to the arrival of eddies along the eastern side of Okinawa could be established from the along-track or mapped satellite altimetry.

[37] Here, rather than comparing the entire Kuroshio and Ryukyu Current transport records, as was done in Andres et al. [2008a] and shown in Figure 2c, we separate periods when cyclones and anticyclones are affecting the two currents as follows. A time series of positive transport anomalies is constructed for the Kuroshio by setting transport to zero when it falls below the mean. Likewise, a time series of negative Kuroshio transport anomalies is constructed by setting transport to zero when it is above the mean. This procedure is repeated for the Ryukyu Current. Figure 15 shows the lagged correlations between Ryukyu Current and Kuroshio transports where the positive and negative transport anomalies have been separated as discussed above. Lagged correlations between Kuroshio positive transport anomalies and Ryukyu Current positive transport anomalies are shown with triangles and lagged correlations between the two currents' negative transport anomalies are shown with squares. The positive transport anomalies have a maximum correlation at 60-day lag. This indicates that an increase in the Ryukyu Current transport at the O-line (i.e., stronger flow towards the northeast) is followed 60 days later by an increase in the Kuroshio transport at the PN-line (i.e., stronger flow towards the northeast). In contrast, the correlation between the negative transport anomalies is maximum at 40-day lag (Figure 15). This indicates that a decrease in the Ryukyu Current transport at the O-line is followed 40 days later by a decrease in the Kuroshio transport at the PN-line. These lags are consistent with the estimates discussed above (Figure 15, dashed and dotted lines) which suggest that positive transport anomalies should be correlated at a longer lag than the negative transport anomalies. In particular, the 40-day lag of the negative transport anomalies is in excellent agreement with the laboratory and scaling estimates, while the estimate of the lag for the positive transport anomalies (90 days) is slightly larger than observed (60 days). Although slightly different values of the eddy diameter and azimuthal velocity may produce better agreement between the estimated lag and that observed for the positive transport anomalies, the above comparison should be considered only as indication that the dynamics observed in the laboratory may be relevant around Okinawa Island. Furthermore, if anticyclones and cyclones were equally likely to impinge on Okinawa, one might expect the overall correlation between the Ryukyu Current and Kuroshio variability (i.e. including both the positive and negative transport anomalies in the comparison) to peak at 50-day lag. However, the correlation between the complete transport time series is maximum at 60-day lag (Figure 2) suggesting that anticyclones are slightly more frequent than cyclones. Indeed, observations corroborate that anticyclones are slightly more common than cyclones in this region [Hwang et al., 2004; Chang and Oey, 2011].

\section{Conclusions}

[38] Experiments investigating the interaction of baroclinic anticyclonic and barotropic cyclonic eddies with a long and narrow island suggest a possible mechanism to explain the lag observed between the transport increase (decrease) in the Ryukyu Current and the transport increase (decrease) in the Kuroshio near Okinawa Island. The laboratory results presented here suggest that the interaction between an eddy and an island is governed by conservation of circulation around the island. The dissipation along the island where the eddy flows is balanced by the dissipation along the island where a streamer flows in the opposite direction. An anticyclone impinging on the island leads to a streamer flowing clockwise around the island, while a cyclone induces a counterclockwise streamer (Figures 13 and 14). Hence, we expect the interaction between an anticyclone (cyclone) and Okinawa Island to result in an instantaneous increase (decrease) of the Ryukyu Current transport and a delayed increase (decrease) of the Kuroshio transport (Figure 13). The lag correlations predicted from the laboratory experiments and scaling analysis compare favorably with the lag correlations obtained with field observations, suggesting that the dynamics described above may be at play around Okinawa Island.

[39] There are clearly significant differences between the laboratory setup and the real ocean. These include simplified topography, simplified eddy vertical structure, and absence of local forcing. Also, unlike the anticyclones generated in the 


\section{ANDRES AND CENEDESE: EDDIES IMPINGING ON AN ISLAND}

laboratory, most eddies in the ocean do not spin down by Okinawa but continue moving southwest along the Ryukyu Island Chain towards Taiwan. Nevertheless, comparison of the laboratory results with field observations from Okinawa is consistent and suggests, to first order, that the process reproduced in the laboratory has an analog in the real ocean. This implies a degree of predictability for the Kuroshio transport variations and for flow through the Kerama Gap, southwest of Okinawa. Further, this mechanism of eddy-island interaction likely is relevant around other islands, such as Taiwan, that are in the path of westward propagating eddies.

[40] Acknowledgments. We wish to thank Anders Jensen for providing invaluable assistance in the laboratory. Helpful suggestions from two anonymous reviewers improved the manuscript. M.A. was supported by the Postdoctoral Scholar Program at the Woods Hole Oceanographic Institution, with funding provided by the Ocean and Climate Change Institute and by the Penzance Endowed Fund in Support of Assistant Scientists.

\section{References}

Adduce, C., and C. Cenedese (2004), An experimental study of a monopolar vortex colliding with cylinders of varying geometry in a rotating fluid, J. Mar. Res., 62, 611-638.

Andres, M., J.-H. Park, M. Wimbush, X.-H. Zhu, K.-I. Chang, and H. Ichikawa (2008a), Study of the Kuroshio/Ryukyu Current system based on satellitealtimeter and in situ data measurements, J. Oceanogr., 64, 937-950, doi:10.1007/s10872-008-0077-2.

Andres, M., M. Wimbush, J.-H. Park, K.-I. Chang, B.-H. Lim, D.R. Watts, H. Ichikawa, and W. J. Teague (2008b), Observations of Kuroshio flow variations in the East China Sea, J. Geophys. Res., 113, C05013, doi:10.1029/2007JC004200.

Cenedese, C., and P. F. Linden (1999), Cyclone and anticyclone formation in a rotating stratified fluid over a sloping bottom, J. Fluid Mech., 381, 199-223.

Cenedese, C. (2002), Laboratory experiments on mesoscale vortices colliding with a seamount, J. Geophys. Res., 107(C6), 3053, doi:10.1029/ 2000JC000599.

Cenedese, C., C. Adduce, and D. M. Fratantoni (2005), Laboratory experiments on mesoscale vortices interacting with two islands, J. Geophys. Res., 110, C09023, doi:10.1029/ 2004JC002734.

Cenedese, C., R. E. Todd, G. G. Gawarkiewicz, W. B. Owens, and A. Y. Shcherbina (2013), Offshore transport of shelf waters through interaction of vortices with a shelfbreak current, J. Phys. Oceanogr., doi:10.1175/ JPO-D-12-099.1, in press.
Chang, Y.-L., and L.-Y. Oey (2011), Interannual and seasonal variations of Kuroshio transport east of Taiwan inferred from 29 years of tide-gauge data, Geophys. Res. Lett., 38, L08603, doi:10.1029/2011GL047062.

Griffiths, R. W., and P. F. Linden (1981), The stability of vortices in a rotating, stratified fluid, J. Fluid Mech., 4, 283-316.

Godfrey, J. S. (1989), A Sverdrup model of the depth-integrated flow from the world ocean allowing for island circulations, Geophys. Astrophys. Fluid Dyn., 45, 89- 112.

Hedstrom, K., and L. Armi (1988), An experimental study of homogeneous lenses in a stratified rotating fluid, J. Fluid Mech., 191, 535-556.

Hwang, C., C.-R. Wu, and R. Kao (2004), TOPEX/Poseidon observations of mesoscale eddies over the STCC, J. Geophys. Res., 109, C08013, doi:10.1029/2003JC002026.

Ichikawa, H., H. Nakamura, A. Nishina, and M. Higashi (2004), Variability of north-eastward current southeast of northern Ryukyu Islands, J. Oceanogr., 60, 351-363.

Lentz, S. J., and K. R. Helfrich (2002), Buoyant gravity currents along a sloping bottom in a rotating fluid, J. Fluid Mech., 464, 251-278.

Konda, M., H. Ichikawa, I.-S. Han, and X.-H. Zhu (2005), Variability of current structure due to meso-scale eddies on the bottom slope southeast of Okinawa Island, J. Oceanogr., 61, 1089-1099.

Kundu, P. K., and L. M. Cohen (1990), Fluid Mechanics, 5th ed., 638 pp., Academic Press, New York.

Ou, H. W., and A. L. Gordon (1986), Spin-Down of baroclinic eddies under sea ice, J. Geophys. Res., 91, C6, 7623-7630.

Pedlosky, J., L. J. Pratt, M. A. Spall, and K. R. Helfrich (1997), Circulation around islands and ridges, J. Mar. Res., 55, 1199-1251.

Qiu, B. (1999), Seasonal eddy field modulation of the North Pacific Subtropical Countercurrent: TOPEX/Poseidon observations and theory, J. Phys. Oceanogr., 29, 2471-2486.

Simpson, J. E. (1997), Gravity Currents in the Environment and the Laboratory, 2nd ed., chapter 11, 140-163 pp., Cambridge University Press, Cambridge.

Tanabe, A., and C. Cenedese (2008), Laboratory experiments on mesoscale vortices colliding with an island chain, J. Geophys. Res., 113, C04022, doi:10.1029/2007JC004322.

Whitehead, J. A., M. E. Stern, G. R. Flierl, and B. A. Klinger (1990), Experimental observations of baroclinic eddies on a sloping bottom, J. Geophys. Res., 95, 9585-9610.

Zhu, X.-H., I.-S. Han, J.-H. Park, H. Ichikawa, K. Murakami, A. Kaneko, and A. Ostrovskii (2003), The northeastward current southeast of Okinawa Island observed during November 2000 to August 2001, Geophys. Res. Lett., 30(2), 1071, doi:10.1029/2002GL015867.

Zhu, X.-H., H. Ichikawa, K. Ichikawa, and K. Takeuchi (2004), Volume transport variability southeast of Okinawa Island estimated from satellite altimeter data, J. Oceanogr., 60, 953-962.

Zhu, X.-H., J.-H. Park, and D. Huang (2008), Observation of baroclinic eddies southeast of Okinawa Island, Science in China. Series D, Earth Sciences, 51(12), doi:10.1007/s11430-008-0146-9. 